\title{
Towards a Quality Model for Choreography^
}

\author{
Michele Mancioppi ${ }^{1}$, Mikhail Perepletchikov ${ }^{2}$, \\ Caspar Ryan ${ }^{2}$, Willem-Jan van den Heuvel ${ }^{1}$, and Mike P. Papazoglou ${ }^{1}$ \\ 1 European Research Institute in Services Science (ERISS), \\ Tilburg University, The Netherlands \\ $\{$ m.mancioppi, wjheuvel, mikep\}@uvt.nl \\ 2 School of Computer Science and IT, \\ RMIT University, Australia \\ \{mikhail.perepletchikov, caspar.ryan\}@rmit.edu.au
}

\begin{abstract}
Quality attributes of software products like maintainability and reliability have been widely studied in the Software Engineering literature. Their understanding has proven instrumental for developing best practices and tooling support that ultimately result in higher-quality software.

In this paper we investigate external quality attributes (i.e. aspects of quality visible to the end user) of service choreographies. Service choreographies are service compositions that specify completely distributed, message-based interactions among services. Our work is a first step towards the definition of a quality model for service choreographies.
\end{abstract}

\section{Introduction}

Service Oriented Architecture (SOA) envisions software services that provide functionalities to each other and to consumers. Service compositions aggregate existing services to create value-added ones. Service choreography (choreography, for brevity) is a service composition paradigm that specifies conversations among the composed services, i.e. its participants, from a global perspective 11. Participants in a choreography are independent agents that interact with each other by exchanging messages. In the practice of SOA, choreographies can be specified with modeling notations like Web Service Choreography Description Language (WS-CDL) and Business Process Modeling Notation (BPMN) v1.2.

One aspect that is missing in the state of the art of choreographies is the "big picture" of what are the relevant quality aspects of choreography specifications. The comprehensive specification and evaluation of appropriate quality attributes is a key factor in ensuring adequate quality in the production of any kind of artifacts like physical goods, software source code, etc. To this end, quality models have been produced over time for a number of general and specialized areas.

\footnotetext{
* The research leading to these results has received funding from the European Community's Seventh Framework Programme under the Network of Excellence S-Cube - Grant Agreement $\mathrm{n}^{\circ}$ 215483. This work is also funded by the ARC (Australian Research Council), under Discovery scheme no. DP0988345.
} 
Quality models are frameworks that provide concrete mechanisms for reasoning about quality in a systematic and objective manner [2]. They should be generic enough to cover the needs of different stakeholders, e.g. architects, designers, developers and end-users.

Quality models are composed of quality attributes and quality metrics. Quality attributes are desirable properties of the artifacts described by the quality model. They represent a checklist of quality concerns, and can be divided into external and internal attributes. External quality attributes describe how the quality of an artifact is perceived by its end users, e.g. in terms of Reliability or Usability. Internal quality attributes describe the quality of the artifact's design or implementation, e.g. in terms of Coupling and Cohesion [3. Quality metrics are measurement mechanisms that quantitatively evaluate and/or predict internal and external quality attributes. While the external quality attributes of an artifact can be fully measured only after its development is completed, they can be predicted by measuring the internal quality attributes. For example, it has been empirically shown that the internal quality attributes of software design have a strong causal impact on external quality attributes in both Procedural [4] and Object-Oriented [56] software products. The prediction of the external quality attributes and the consequent improvement early in the Software Development LifeCycle (SDLC) results in a higher-quality software products 78 .

Our goal is to unlock the benefits of a systematic and comprehensive quality management process for choreography specifications - i.e. descriptions of the structure of choreographies - by working towards a dedicated quality model. To this end, this paper presents our preliminary findings on the external quality attributes of choreographies. Our work is intended to complement the ongoing research work on quality attributes for choreography modeling notations (i.e. the syntaxes used to model choreographies) such as [9. An overview of choreography and an outline of its aspects that must be considered for the formulation of the quality attributes is presented in Section 2, Section 3 presents an initial suite of external quality attributes specific to choreography. Finally, Section 4 concludes the paper by presenting our closing remarks and future directions.

\section{Choreographies for Service-Oriented Architecture}

This section presents the background knowledge on choreographies that we find relevant for the formulation of choreography quality attributes: their usage in SOA (Section 2.1), the elements they are made of (Section 2.2), and which of their properties are usually verified (Section 2.3). Due to space constraints we reference the interested reader to [1011] for more information on the development and lifecycle of the choreographies.

\subsection{Usage of Choreographies}

Before treating what choreographies are used for in SOA, it is necessary to underline what choreographies are not used for: direct execution on the service 
infrastructure. Unlike orchestrations, for example a Business Process Execution Language (BPEL) process deployed on and run by a BPEL engine, choreographies are not deployed on engines to be executed. A choreography is a service composition whose logic is distributed across and shared by its participants. The participants are independent agents (e.g. software services) possibly owned and run by different organizations. At run-time, each participant is responsible for the correct execution of its role in the choreography, i.e. its expected messaging behavior, in conjunction with what the other participants are doing. When the participants execute their roles in concert, they enact the choreography.

In the practice of SOA, choreographies are used for:

Specification and communication: choreographies are used to specify and communicate to the participants the requirements on the messaging interactions that they must fulfill, i.e. their roles.

Facilitating the implementation of the participants: during the process of implementing the participants (or the evolution of existing participants to fit a new choreography), the choreography can be used to generate (1) participant skeletons (i.e. partial implementations, see for example [12) and (2) test cases for the participant implementations [13].

Monitoring the enactments: the monitoring facilities (e.g. integrated in the Enterprise Service Bus [14]) verify that the enactments do not violate the choreography.

\subsection{Choreography Elements}

The modeling notations for choreography (called in the remainder "choreography notations" or simply "notations" for brevity) vary greatly in the syntaxes and constructs they provide. Some have only an eXtensible Markup Language (XML) representation, e.g. WS-CDL. Others are graphical notations, e.g. Let's Dance [15]. Regardless of the syntactical differences among the various choreography notations, the constructs they provide can be categorized as follows:

Message ordering constructs define the ordering of the message exchanges that take place among the participants. Message exchanges are usually specified as (1) the identifier of the message, (2) the identifiers of the participant that sends the message (the sender) and of one or more participants that receive it (the recipients), and possibly (3) time constraints (see for example [1617]). No technical information such as participants' endpoints and interfaces or message types is provided at this level.

Message types constructs describe the structure of the messages that are exchanged among the participants, e.g. using XML Schema.

Grounding constructs provide the technical details necessary to enact the choreography, e.g. the endpoints of the participants and which transport protocols are supported to exchange messages (e.g. SOAP over HTTP).

Every choreography notation provides message ordering constructs. However, message types and grounding constructs are provided only by some (e.g. WS-CDL). 
Another important aspect of a choreography notation is the messaging model it assumes, namely synchronous or asynchronous. In the synchronous case, the recipients must be ready to accept inbound messages when the sender delivers them. In asynchronous messaging, messages are posted by the sender and stored in queues held by the recipients. Each recipient can then decide if and when to consume the messages stored in its queue. Different types of asynchronous messaging make different assumptions on the size of the queues (finite or unbound), expiration time of the messages (a message is automatically removed from a queue if not consumed within a certain amount of time), preservation of message orders in the queues (whether the messages are stored in the queue in the same order in which they are sent), etc.

\subsection{Properties of Choreographies}

The distribution inherent to choreographies makes them susceptible to defects like deadlocks and the specification of roles that can not be faithfully implemented by the participants. Consequently, much effort has been devoted to the investigation of desirable properties such as deadlock freeness and realizability. In this section we briefly introduce the most relevant of these properties, providing the interested reader with references to more exhaustive material.

Deadlock Freeness. A deadlock occurs when the enactment of a choreography reaches a state that (1) is not final and (2) can not be left without violating the message ordering of the choreography. Analyses of choreographies for deadlock freeness usually build on top of model checking techniques (e.g. [18]) or on structural properties of the adopted choreography notation (e.g. [19]).

Conformance. A participant conforms to a choreography if its business protocol (i.e. the actual messaging behavior of the participant as perceived by the others [2017]) is equivalent to the role played by that participant in the choreography. The actual notion of equivalence varies across different approaches in the literature, ranging from language equivalence - the business protocol and the role can execute all and only the same traces (see for example 21]) - to bisimulation (see e.g. [22])

Realizability. Not all choreographies that can be specified can actually be correctly implemented by their participants. In some cases it is impossible for the participants to provide implementations that conform to the choreography. This problem occurs when a choreography specifies message exchanges between the participants that do not provide them with enough information on the global state of the enactments. The lack of information can lead the participants to unknowingly violate the choreography. In this case, the choreography is said to be not realizable 231] (a.k.a. enactable or enforceable).

Realizability is a paramount property of choreographies. Only realizable choreographies can really be enacted correctly in a completely distributed manner. It has been shown that non-realizable choreographies can be made realizable by further adding message exchanges that, during the enactment, make the participants align their perception on the global state [24]. 


\section{Choreography Quality Attributes}

This section presents our initial formulation of the external quality attributes for choreography. We focus on the quality of two aspects of a choreography: the choreography as a specification and how they way it is specified affects its enactments. Concerning the elements of choreographies (see Section 2.2), we limit ourselves to quality attributes related to the message type and message ordering constructs. We do not take into account the grounding of choreographies because the quality attributes it induces are closely related to the quality of the individual participant implementations (e.g. in terms of their reliability, availability, and trustworthiness [25[26]). Nonetheless, we understand the importance of the grounding in the context of choreography quality and plan to investigate this area in future work.

The external quality attributes we present and they way they are hierarchically organized in main- and sub-attributes are partly inspired by ISO/IEC 9126-1 2]. Their formulation is based on an extensive literature review about choreographies and other quality models focusing on different, but related areas like web services and Service-Based Applications (SBAs).

Similarly to ISO/IEC 9126-1, all the main attributes include "compliance" sub-attributes, e.g. Security Compliance. Compliance sub-attributes capture the capability of the choreography to comply with standards, conventions, regulations, organizational policies and similar prescriptions that concern their main attributes. The particular prescriptions a choreography must comply with depend on the context of the definition of the choreography and of its enactments. For example, Security Compliance might capture the adherence of a certain choreography to the data privacy policies of the organizations that participate in it, as well as the compliance with regulations on data encryption and anonymization. All compliance sub-attributes are conceptually the same, and thus their definition under each attribute is omitted in the remainder.

The remainder of this section provides a detailed description of the external quality attributes and sub-attributes separately.

\section{$3.1 \quad$ Functionality}

Functionality describes the quality of a choreography as a specification, i.e. how appropriate is the choreography to specify and communicate to the participants the requirements in terms of messaging interactions (see also Section 2.1).

Suitability is the capability of the choreography to satisfy its stated requirements in terms of message interactions among the participants, and how the message interactions make them achieve the desired goals.

Accuracy is the capability of the choreography during its enactments to make its participants achieve the correct or agreed results or effects with the needed degree of closeness. For example, the enactment of an accurate choreography will meet the business goals shared by the participants in an amount of time that is suitable for them (e.g. under one hour for the shipment of an online order). 
Understandability and Completeness are related to the interpretation of the choreography as a specification by its participants. Understandability is the capability of the choreography to be univocally understood by its intended audience, e.g. the developers in charge of implementing the participants. Completeness is the capability of a choreography not to leave un- or underspecified aspects that, left open to the interpretation of the participants, might result in incompatibilities.

Reusability is the capability of a choreography specification to be reused in whole or in part for specifying other messaging interactions. The reuse of choreographies can reduce the cost implementing the participants, for example by facilitating the reuse of source code and test cases.

\section{$3.2 \quad$ Security}

Security is the capability of the choreography to prevent corruption and unauthorized access to information and data during both the implementation of the participants and the enactments.

Confidentiality captures the need of participants to (1) exchange privately sensitive data and information during the enactments of the choreography and (2) do not divulge sensitive information in the choreography specification.

Non-Repudiation and Authenticity are both facets of the issue of ensuring the identities of the participants in a choreography. Non-Repudiation is the capability of the choreography of preventing participants from refuting or repudiating messages they have sent or received during the enactments of a choreography (e.g. by using digital signatures and reception acknowledgements). Authenticity refers to the guarantees that the choreography specification provides in terms of authentication of messages, i.e. that the message actually comes from its supposed sender and not from another entity.

Message Integrity is the capability of the choreography to ensure the integrity of the messages that are exchanged by the participants during the enactments, e.g. by requiring mechanisms for detecting corrupted messages (for example hashing of the messages' payloads) and having them sent again.

Finally, Message Reliability deals with the level of reliability set by the choreography for the message exchanges. It should be noted that the actual implementation of the reliability mechanisms is usually delegated to the middleware employed by the participant implementations.

\section{$3.3 \quad$ Efficiency}

Efficiency is the capability of the choreography to specify interactions among the participants so that the enactments efficiently achieve the choreography's goals relative to the amount of resources used. The actual design of a choreography influences the efficiency of its enactments, e.g. in terms of average completion time. Aspects of efficiency of choreographies have been investigated under the umbrella of Quality of Service (QoS) (e.g. 27]). As anticipated at the beginning of the section, we do not consider the grounding of choreographies. On the basis 
of the message type and ordering, we have identified two sub-attributes of efficiency of choreographies: Message Type Efficiency and Message Ordering Efficiency.

Message Type Efficiency relates the amount of data and information that are conveyed over message exchanges with the formatting of the messages (i.e. how the message types are specified) and the computing and bandwidth resources that are consumed to perform the message exchanges and process the messages (e.g. composition and compression by the sender, decompression and parsing by the recipients).

Message Ordering Efficiency captures how the ordering of the message exchanges in the choreography affects (1) the amount of time necessary to complete its enactments, and (2) the involvement (in terms of amounts of time) of the participants in the enactments.

\subsection{Implementability}

Implementability is the capability of the choreography to support and facilitate the implementation of its participants and their interactions during the enactments. The design and specific properties of a choreography influence how its participants are implemented.

Realizability captures the fact that the choreography is specified such that its participants can be implemented so to conform with the required messaging behaviors (see Section 2.3).

Projectability and Testability are related to how the choreography facilitates and supports the use of tools, possibly through Integrated Development Environments (IDEs), to simplify the implementation of its participants. The Projectability is the capability of choreography to be used to generate skeletons of the participant implementations (see Section 2.1). Testability is the capability of a choreography to be used to generate test cases for the participant implementations (see Section 2.1).

Deadlock Freeness refers to the absence of deadlocks in the enactments of the choreography (see Section 2.3).

Monitorability of a choreography refers to how its design affects the monitoring of its enactments (see Section 2.1). For example, the fact that each participant has only partial information during the enactment may complicate the monitoring. Participants that need to monitor parts of the choreography enactment might agree to share with each other key monitoring events.

\subsection{Maintainability}

Choreographies are specifications, and specifications are changed over time to reflect how their requirements evolve. Maintainability is the capability of the choreography to be changed over time to meet its evolving functional and nonfunctional requirements, e.g. by introducing or removing participants and message exchanges. We believe that the existing taxonomies of Software Maintenance (e.g. 28]) are largely applicable to choreography specifications. 
Analyzability is the capability of a choreography to be analyzed using automatic tools like model checkers, simulators, and performance analyzers. The size and complexity of the choreography (e.g. amount of parallelism) affects the feasibility of most analysis methods because of the problem of state explosion. Moreover, the formality and expressiveness of the adopted notation could affect the analyzability of the choreographies modeled with it.

Changeability is the capability of the choreography to be modified with minimal effort and predictable results in terms of change propagation, i.e. how changes to the modified parts "ripple through" the choreography and affect the unmodified ones. Similarly to traditional software products, choreographies with a complicated and highly-coupled structure (i.e. message ordering) are likely harder to modify than simpler, more streamlined ones.

Versionability is the capability of the choreography to be clearly identified in the scope of version control, i.e. the management of different versions of the choreography that are produced during maintenance. Versionability can be defined at both specification- and enactment level. Specification-level versionability of a choreography is its capability of conveying information about its versioning in terms of (1) which other choreography specification it originates from (i.e. its baseline), (2) which changes have been applied to the baseline to lead to this version, and (3) when those changes have been applied, either in terms of their order or (better) the timestamp of their application. Alternatively, this can be achieved using revision control systems like SVN and GIT.

Enactment versionability is the capability of the choreography to have its version identified during its enactments. Participants need to identify the version of the choreography during its enactments in order to avoid issues resulting from the lack of backward- and forward compatibility across versions. A straightforward way of achieving this is embedding the choreography's version identifier in the messages exchanged by the participants. More elaborate ways might be based on the related work on business process versionability, see for example [29].

It should be noted that, in addition to the proposed maintainability subattributes of choreographies, the process of maintaining a choreography also may depend on some of the sub-attributes of Functionality, namely Understandability and Reusability.

\section{Conclusions and Future Work}

In this work we have proposed our initial findings concerning external quality attributes for service choreographies, i.e. their quality aspects as perceived by the end users. Service choreography is a paradigm of service composition that specifies distributed, message-based interactions among multiple participants.

There is no work in the state of the art on quality models for choreographies. This paper takes a first step in that direction. We have overviewed the aspects of service choreography that are relevant for the definition of its quality model: usage in the practice of SOA, constructs, and verification. On the basis of this overview, we have proposed a hierarchy of external quality attributes that cover 
five main quality aspects of choreographies: functionality as specifications, security, efficiency, implementability (i.e. the aspects of choreographies that influence how the participants are implemented), and maintainability.

Currently, we are considering different approaches for validating the proposed external quality attributes and sub-attributes. Additionally, we foresee several ways of extending the proposed model of external quality attributes for choreographies, for example the inclusion of attributes related to the grounding of the choreographies, costs and revenues sustained by the participants, and classical non-functional properties of distributed systems like recoverability, fault tolerance, and transactionality.

Finally, it is our intention to investigate the structural properties (or internal quality attributes) of choreographies (e.g. cohesion, coupling, and complexity) and define associated metrics for quantifying these properties in an objective and automated manner. Such metrics can be based on established metrics from the traditional software engineering field and the growing body of work on metrics for business processes.

\section{References}

1. Su, J., Bultan, T., Fu, X., Zhao, X.: Towards a theory of web service choreographies. In: Dumas, M., Heckel, R. (eds.) WS-FM 2007. LNCS, vol. 4937, pp. 1-16. Springer, Heidelberg (2008)

2. ISO/EIC 9126-1:2001: Software engineering - Product quality - Part 1: Quality model. International Organization for Standardization, Geneva, Switzerland (2001)

3. Perepletchikov, M., Ryan, C., Frampton, K., Schmidt, H.W.: Formalising serviceoriented design. JSW 3(2), 1-14 (2008)

4. Henry, S.M., Selig, C.: Predicting source-code complexity at the design stage. IEEE Software 7(2), 36-44 (1990)

5. Briand, L.C., Wüst, J., Daly, J.W., Porter, D.V.: A comprehensive empirical validation of design measures for object-oriented systems. In: IEEE METRICS, pp. 246-257. IEEE Computer Society, Los Alamitos (1998)

6. Alshayeb, M., Li, W.: An empirical validation of object-oriented metrics in two different iterative software processes. IEEE Trans. Software Eng. 29(11), 10431049 (2003)

7. Perepletchikov, M., Ryan, C., Frampton, K.: Cohesion metrics for predicting maintainability of service-oriented software. In: QSIC, pp. 328-335. IEEE Computer Society, Los Alamitos (2007)

8. Perepletchikov, M., Ryan, C., Frampton, K., Tari, Z.: Coupling metrics for predicting maintainability in service-oriented designs. In: ASWEC, pp. 329-340. IEEE Computer Society, Los Alamitos (2007)

9. Decker, G.: Design and analysis of process choreographies. PhD thesis, Hasso Plattner Institute, University of Potsdam (June 2009)

10. Decker, G., Riegen, M.V.: Scenarios and techniques for choreography design. In: Abramowicz, W. (ed.) BIS 2007. LNCS, vol. 4439, pp. 121-132. Springer, Heidelberg (2007)

11. Decker, G., Kopp, O., Barros, A.P.: An introduction to service choreographies (Servicechoreographien - eine Einführung). it - Information Technology 50(2), 122 127 (2008) 
12. Mendling, J., Hafner, M.: From inter-organizational workflows to process execution: Generating BPEL from WS-CDL. In: Meersman, R., Tari, Z., Herrero, P. (eds.) OTM-WS 2005. LNCS, vol. 3762, pp. 506-515. Springer, Heidelberg (2005)

13. Wieczorek, S., Roth, A., Stefanescu, A., Charfi, A.: Precise steps for choreography modeling for SOA validation and verification. In: SOSE, pp. 148-153. IEEE Computer Society, Los Alamitos (2008)

14. Kopp, O., van Lessen, T., Nitzsche, J.: The need for a choreography-aware service bus. In: YR-SOC 2008, pp. 28-34 (June 2008) (Online)

15. Zaha, J.M., Barros, A.P., Dumas, M., ter Hofstede, A.H.M.: Let's Dance: A language for service behavior modeling. In: Meersman, R., Tari, Z. (eds.) OTM 2006. LNCS, vol. 4275, pp. 145-162. Springer, Heidelberg (2006)

16. Kazhamiakin, R., Pandya, P.K., Pistore, M.: Timed modelling and analysis in web service compositions. In: ARES, pp. 840-846. IEEE Computer Society, Los Alamitos (2006)

17. Mancioppi, M., Carro, M., van den Heuvel, W.J., Papazoglou, M.P.: Sound multiparty business protocols for service networks. In: Bouguettaya, A., Krueger, I., Margaria, T. (eds.) ICSOC 2008. LNCS, vol. 5364, pp. 302-316. Springer, Heidelberg (2008)

18. Lohmann, N., Kopp, O., Leymann, F., Reisig, W.: Analyzing BPEL4Chor: Verification and participant synthesis. In: Dumas, M., Heckel, R. (eds.) WS-FM 2007. LNCS, vol. 4937, pp. 46-60. Springer, Heidelberg (2008)

19. Qiu, Z., Zhao, X., Cai, C., Yang, H.: Towards the theoretical foundation of choreography. In: WWW, pp. 973-982. ACM, New York (2007)

20. Ponge, J., Benatallah, B., Casati, F., Toumani, F.: Fine-grained compatibility and replaceability analysis of timed web service protocols. In: Parent, C., Schewe, K.D., Storey, V.C., Thalheim, B. (eds.) ER 2007. LNCS, vol. 4801, pp. 599-614. Springer, Heidelberg (2007)

21. Baldoni, M., Baroglio, C., Martelli, A., Patti, V., Schifanella, C.: Verifying the conformance of web services to global interaction protocols: A first step. In: Bravetti, M., Kloul, L., Zavattaro, G. (eds.) EPEW/WS-EM 2005. LNCS, vol. 3670, pp. 257-271. Springer, Heidelberg (2005)

22. Busi, N., Gorrieri, R., Guidi, C., Lucchi, R., Zavattaro, G.: Choreography and orchestration conformance for system design. In: Ciancarini, P., Wiklicky, H. (eds.) COORDINATION 2006. LNCS, vol. 4038, pp. 63-81. Springer, Heidelberg (2006)

23. Fu, X., Bultan, T., Su, J.: Realizability of conversation protocols with message contents. Int. J. Web Service Res. 2(4), 68-93 (2005)

24. Salaün, G., Bultan, T.: Realizability of choreographies using process algebra encodings. In: Leuschel, M., Wehrheim, H. (eds.) IFM 2009. LNCS, vol. 5423, pp. 167-182. Springer, Heidelberg (2009)

25. Ran, S.: A model for web services discovery with QoS. SIGecom Exchanges 4(1), $1-10(2003)$

26. Gehlert, A., Metzger, A.: Quality reference model for SBA. Contractual Deliverable CD-JRA-1.3.2, S-Cube Consortium (March 2009), http://www.s-cube-network.eu/results/deliverables

27. Zhao, X., Cai, C., Yang, H., Qiu, Z.: A QoS view of web service choreography. In: ICEBE, pp. 607-611. IEEE Computer Society, Los Alamitos (2007)

28. Chapin, N., Hale, J.E., Khan, K.M., Ramil, J.F., Tan, W.G.: Types of software evolution and software maintenance. Journal of Software Maintenance 13(1), 3-30 (2001)

29. Juric, M.B., Sasa, A., Rozman, I.: WS-BPEL extensions for versioning. Information and Software Technology 51(8), 1261-1274 (2009) 